\title{
THE SUPPORTS OF INFINITELY DIVISIBLE DISTRIBUTION FUNCTIONS
}

\author{
HOWARD G. TUCKER
}

ABSTRACT. An infinitely divisible probability measure over the real line whose associated Lévy spectral measure gives nonzero mass to every deleted neighborhood of the origin is shown to have as its support an interval of the form $(-\infty, a],[a, \infty)$ or $(-\infty, \infty)$.

1. Introduction. This is the beginning of a study of the supports of inf $\mathrm{i}$ nitely divisible distribution functions. By the support of a distribution function $F$, supp $F$, we mean the smallest closed set $S$ for which $\int_{S} d F(x)=1$. In a recent paper [4] it was shown that if $F$ is infinitely divisible, without Gaussian component and whose Lévy spectral measure is absolutely continuous with respect to Lebesgue measure and is infinite, then the support of $F$ is an interval of the form $(-\infty, a],[a, \infty)$ or $(-\infty, \infty)$, and that the density of $F$ is positive almost everywhere over its support. In this note it is shown that a relaxed hypothesis on the Lévy spectral measure $M$, namely, $M((-\epsilon, 0) \cup(0, \epsilon))>0$ for all $\epsilon>0$, yields the same conclusion about the support of $F$.

The representation that will be used here for the characteristic function $f(u)$ of an infinitely divisible distribution function $F(x)$ is given by

$$
f(u)=\exp \left\{i \gamma u-\sigma^{2} u^{2} / 2+\int_{x \neq 0}\left(e^{i u x}-1-\frac{i u x}{1+x^{2}}\right) M(d x)\right\},
$$

where $\gamma$ and $\sigma^{2} \geq 0$ are constants and where $M(x)$, the Lévy spectral measure, is a nondecreasing function over $(-\infty, 0)$ and over $(0, \infty)$, satisfying $M(-\infty)=M(+\infty)=0$ and $\int_{-1}^{-0}+\int_{+0}^{1} x^{2} M(d x)<\infty$. In what follows, the same symbol will be used for a distribution function (or any nondecreasing function) and the Lebesgue-Stieltjes measure it determines, i.e., if $G$ is a distribution function, then $G(x)=G((-\infty, x])$, and $G(A)=\int_{A} d G(x)$.

Received by the editors March 22, 1974.

AMS (MOS) subject classifications (1970). Primary 60E05.

Key words and phrases. Support of a distribution function, infinitely divisible distribution function, Lévy spectral measure, independent random variables, distribution function, characteristic function. 
2. The theorem. The sole purpose of this note and this section is to state and prove the following result.

Theorem. If $F$ is an infinitely divisible distribution function with characteristic function given by (1) such that $M((-\epsilon, 0) \cup(0, \epsilon))>0$ for every $\epsilon>0$, then the support of $F$ is an interval of the form $(-\infty, a],[a, \infty)$ or $(-\infty, \infty)$.

Proof. In case $\sigma^{2}>0$, then supp $F=(-\infty, \infty)$; we need therefore only prove the theorem in case $\sigma^{2}=0$. We prove it in three cases.

Case (i). In this case, it is assumed that $F$ has a characteristic function of the form

$$
f(u)=\exp \int_{0}^{\infty}\left(e^{i u x}-1\right) M(d x)
$$

where by hypothesis $M((0, \epsilon))>0$ for all $\epsilon>0$ and $\int_{+0}^{1} x M(d x)<\infty$. By Theorem 1 in [5], if $X$ is a random variable with distribution function $F$, then ess inf $X=0$ and $\sup X=\infty$. We show that if $S=\operatorname{supp} F$, then $S=[0, \infty)$. Suppose not. Then $S$ being closed implies $[0, \infty) \backslash S$ is open and nonempty. Let $(a, b)$ be any nonempty open interval in $[0, \infty) \backslash S$. Since $M((0, \epsilon))>0$ for all $\epsilon>0$, there exists a sequence $x_{n} \downarrow 0$ such that each $x_{n}$ is a point of increase of $M$, i.e., for every $\delta>0, M\left(x_{n}+\delta\right)-M\left(x_{n}-\delta\right)>0$ for all $n$. Hence, there exists an integer $N$ such that $0<x_{N}<b-a$. Let $k$ be a positive integer such that $a<k x_{N}<b$. Let $0<\delta_{0}<x_{N} / 2$, and let $F_{1}$ and $F_{2}$ be two distribution functions whose corresponding characteristic functions are

$$
f_{1}(u)=\exp \left\{\int_{+0}^{x} N^{-\delta_{0}}+\int_{x_{n}+\delta_{0}}^{\infty}\right\}\left(e^{i u x}-1\right) M(d x)
$$

and

$$
f_{2}(u)=\exp \int_{x_{N}-\delta_{0}}^{x} N^{+\delta_{0}}\left(e^{i u x}-1\right) M(d x)
$$

We now show that $F_{2}((a, b))>0$. Let us denote $\lambda=M\left(x_{N}+\delta_{0}\right)-M\left(x_{N}-\delta_{0}\right)$ $>0$, and define

$$
\begin{aligned}
G(x) & =0 \quad \text { if } x<x_{N}-\delta_{0}, \\
& =\left(M(x)-M\left(x_{N}-\delta_{0}\right)\right) / \lambda \quad \text { if } x_{N}-\delta_{0} \leq x<x_{N}+\delta_{0}, \\
& =1 \quad \text { if } x>x_{N}+\delta_{0},
\end{aligned}
$$

where $M$ and $G$ are taken as right continuous. Let $\delta>0$ be such that $a<k\left(x_{N}-\delta\right)<k\left(x_{N}+\delta\right)<b$. Now $G\left(\left(x_{N}-\delta, x_{N}+\delta\right)\right)>0$ since $G$ has a 
point of increase at $x_{N}$. Letting $\left\{X_{1}, \cdots, X_{k}\right\}$ be independent, identically distributed random variables with common distribution function $G$, we have

$$
\begin{aligned}
0 & <\left(G\left(x_{N}+\delta\right)-G\left(x_{N}-\delta\right)\right)^{k}=\prod_{j=1}^{k} P\left[X_{j} \in\left(x_{N}-\delta, x_{N}+\delta\right)\right] \\
& =P\left(\bigcap_{j=1}^{k}\left[x_{j} \in\left(x_{N}-\delta, x_{N}+\delta\right)\right]\right) \leq P\left[\sum_{j=1}^{k} X_{j} \in\left(k\left(x_{N}-\delta\right), k\left(x_{N}+\delta\right)\right)\right] \\
& =G^{* k}\left(\left(k\left(x_{N}-\delta\right), k\left(x_{N}+\delta\right)\right)\right) \leq G^{* k}((a, b))
\end{aligned}
$$

thus proving that $G^{* k}((a, b))>0$, where $G^{* k}$ denotes the $k$-fold convolution of $G$ with itself. But, as is well known,

$$
F_{2}((a, b))=\sum_{n \geq 0} e^{-\lambda} \frac{\lambda^{n}}{n !} G^{* n}((a, b)) \geq e^{-\lambda} \frac{\lambda^{k}}{k !} G^{* k}((a, b))>0,
$$

thus proving $F_{2}((a, b))>0$. Now let $U_{1}$ and $U_{2}$ be two independent random variables whose distribution functions are $F_{1}$ and $F_{2}$ respectively. Since $x_{N}-\delta_{0}>0$, then by Theorem 1 in [5], ess inf $U_{1}=0$. Let $0<\delta_{1}<$ $1 / 2 \max \left\{b-k x_{N}, k x_{N}-a\right\}$. Now $F_{1}\left(\left[0, \delta_{1}\right)\right)>0$. In proving $F_{2}((a, b))>0$ above, we actually proved

$$
F_{2}\left(\left(k x_{N}-\delta_{1}, k x_{N}+\delta_{1}\right)\right)>0 .
$$

Thus,

$$
\begin{aligned}
0 & <F_{1}\left(\left[0, \delta_{1}\right)\right) F_{2}\left(\left(k x_{N}-\delta_{1}, k x_{N}+\delta_{1}\right)\right) \\
& =P\left[U_{1} \in\left[0, \delta_{1}\right)\right] P\left[U_{2} \in\left(k x_{N}-\delta_{1}, k x_{N}+\delta_{1}\right)\right] \\
& =P\left(\left[U_{1} \in\left[0, \delta_{1}\right)\right]\left[U_{2} \in\left(k x_{N}-\delta_{1}, k x_{N}+\delta_{1}\right)\right]\right) \\
& \leq P\left[U_{1}+U_{2} \in\left(k x_{N}-\delta_{1}, k x_{N}+2 \delta_{1}\right)\right] \\
& \leq P\left[U_{1}+U_{2} \in(a, b)\right]=F((a, b)) .
\end{aligned}
$$

This contradicts the fact that $(a, b) \subset[0, \infty) \backslash S$, thus proving the theorem in Case (i).

Case (ii). Assume now that $F$ has the characteristic function

$$
f(u)=\exp \int_{+0}^{\infty}\left(e^{i u x}-1-\frac{i u x}{1+x^{2}}\right) M(d x), \quad \text { where } \int_{+0}^{1} x M(d x)=\infty .
$$

We shall show in this case that $\operatorname{supp} F=(-\infty,+\infty)$. As in [4], we define a 
measure $M_{1}$ by

$$
\begin{aligned}
M_{1}((x, \infty)) & =M((x, \infty)) \quad \text { if } x \geq 1, \\
& =M((1, \infty))+\int_{x}^{1} t M(d t) \text { if } 0<x<1 .
\end{aligned}
$$

As was shown in [4], $M_{1}$ is a Lévy spectral measure such that $\int_{+0}^{1} x M_{1}(d x)$ $<\infty$ and such that $M_{1}((0,1))=\infty$, and hence $M_{1}$ satisfies the hypothes is of Case (i). Let $M_{2}=M-M_{1}$. As in the proof of Theorem 2 in [4], $M_{2}$ is a Lévy spectral measure, and $\int_{+0}^{1} x M_{2}(d x)=\infty$. Now let $F_{1}$ and $F_{2}$ be two infinitely divisible distribution functions whose corresponding characteristic functions are given by

$$
f_{1}(u)=\exp \left\{\int_{0}^{\infty}\left(e^{i u x}-1\right) M_{1}(d x)\right\}
$$

and

$$
f_{2}(u)=\exp \left\{\int_{0}^{1}\left(e^{i u x}-1-\frac{i u x}{1+x^{2}}\right) M_{2}(d x)-i u \int_{1}^{\infty} \frac{x}{1+x^{2}} M_{1}(d x)\right\} .
$$

Then $F=F_{1} * F_{2}$. Applying Case (i) to $F_{1}$, we obtain supp $F_{1}=[0, \infty)$. By Theorem 1 in [5], $F_{2}$ is the distribution function of a random variable which is unbounded in both directions. In order to show that supp $F=(-\infty,+\infty)$, it is sufficient to show that if $a<b$ are arbitrary, then $F((a, b))>0$. Since $F_{2}(x)>0$ for all $x$, there exists a point of increase $x_{2}$ of $F_{2}$ such that if $x_{1}$ is defined by $x_{1}=(a+b) / 2-x_{2}$, then $x_{1}>0$. From Case (i), $x_{1}$ is a point of increase of $F_{1}$. Thus, by an easy argument, $x_{1}+x_{2}=(a+b) / 2$ is a point of increase of $F_{1} * F_{2}$, which implies $F((a, b))>0$, thus proving the theorem in Case (ii).

Case (iii). The general case. If

$$
\int_{0<|x|<1}|x| M(d x)=\infty,
$$

then by Case (ii), supp $F=(-\infty,+\infty)$. So we must consider the case where

$$
\int_{0<|x|<1}|x| M(d x)<\infty \text {. }
$$

Let $F_{0}, F_{1}$ and $F_{2}$ be distribution functions whose corresponding characteristic functions are

$$
\begin{aligned}
& f_{0}(u)=e^{i u \gamma}, \quad \text { where } \gamma=-\int_{-\infty}^{\infty} \frac{x}{1+x^{2}} M(d x), \\
& f_{1}(u)=\int_{-\infty}^{-0}\left(e^{i u x}-1\right) M(d x), \quad f_{2}(u)=\int_{+0}^{\infty}\left(e^{i u x}-1\right) M(d x) .
\end{aligned}
$$


If either $M((-\infty, 0))=0$ or $M((0, \infty))=0$, then by Case (i), we have the conclusion of the theorem, where

$$
\operatorname{supp} F=\operatorname{supp} F_{1} * F_{0}=(-\infty, \gamma]
$$

if $M((0, \infty))=0$, and

$$
\operatorname{supp} F=\operatorname{supp} F_{2} * F_{0}=[\gamma, \infty)
$$

if $M((-\infty, 0))=0$. If $M((-\infty, 0)) M((0, \infty))>0$, then either supp $F_{2} * F_{0}=$ $[\gamma, \infty)$ and $F_{1}(x)>0$ for all $x$, which implies $\operatorname{supp} F=(-\infty, \infty)$, or supp $F_{1} * F_{0}=(-\infty, \gamma]$ and $F_{2}(x)<1$ for all $x$, which also implies supp $F=$ $(-\infty,+\infty)$ Q.E.D.

From the proof given above, one can easily compute the value of the constant $a$ in the conclusion of the theorem for the various cases that arise.

Corollary. If $F$ is a continuous infinitely divisible distribution function, then the support of $F$ is always of the forms $(-\infty, \infty),(-\infty, a]$ or $[a, \infty)$.

Proof. By a theorem due to Blum and Rosenblatt [1], Hartman and Wintner [3] and Doeblin [2], $F$ is continuous if and only if $\sigma^{2}>0$ or

$$
M((-\epsilon, 0) \cup(0, \epsilon))=\infty \text { for all } \epsilon>0 .
$$

Thus the hypothesis of the theorem above is satisfied, thus yielding the conclusion.

\section{REFERENCES}

1. J. R. Blum and M. Rosenblatt, On the structure of infinitely divisible distributions, Pacific J. Math. 9 (1959), 1-7. MR 21 \#4665.

2. W. Doeblin, $S_{u r}$ les sommes d'un grands nombres des variables aléatoires indépendentes, Bull. Sci. Math. 63 (1939), 23-32, 35-64.

3. P. Hartman and A. Wintner, On the infinitesimal generators of integral convolutions, Amer. J. Math. 64 (1942), 273-298. MR 4, 18.

4. W. N. Hudson and H. G. Tucker, Equivalence of infinitely divisible distributions, Ann. Probability (to appear).

5. H. G. Tucker, Best one-sided bounds for infinitely divisible random variables, Sankhyā Ser. A 23 (1961), 387-396. MR 25 \#3558.

DEPARTMENT OF MATHEMATICS, UNIVERSITY OF CALIFORNIA AT IRVINE, IRVINE, CALIFORNIA 92664 